\title{
然
}

Miguel Casero*

Clara Crespo*

María Mateo*

Jesús Vidal*

\section{EL PAPEL DEL FONDO MONETARIO INTERNACIONAL EN AMÉRICA LATINA DURANTE LA CRISIS DEL CORONAVIRUS}

La crisis desencadenada por la COVID-19 está suponiendo un duro golpe para los países de América Latina. A la factura humana cobrada por la pandemia se suma un fuerte impacto sobre el tejido económico y social. El estancamiento del crecimiento de la región en los últimos años, así como las características socioeconómicas de muchos de estos países, con altos niveles de desigualdad e importantes bolsas de pobreza (exacerbados por la pandemia) ponen en riesgo los éxitos cosechados en la etapa previa. En este contexto, este artículo repasa la actividad financiera desplegada por el Fondo Monetario Internacional y su compromiso en la región y se analizan las posibilidades que ofrece una probable nueva asignación general de derechos especiales de giro por parte del FMI para apoyar a países de renta media con mucha población por debajo de la línea de la pobreza.

Palabras clave: COVID-19, FMI, pobreza, crisis, derechos especiales de giro.

Clasificación JEL: I38, O54.

\section{Introducción}

La historia del Fondo Monetario Internacional (FMI) en América Latina es una historia de desconfianza mutua, la de una relación entre

* Técnico Comercial y Economista del Estado, destinado en la Subdirección General de Relaciones con el Fondo Monetario Internacional y el G20.

Las opiniones de los autores no representan necesariamente la posición de la Secretaría General del Tesoro y Financiación Internacional.

Los autores agradecen los comentarios de Pablo Moreno y Rosa Moral.

Versión de marzo 2021.

DOI: https:/doi.org/10.32796/bice.2021.3133.7176 acreedor y deudor animada, a la vez que contaminada, por las decisiones políticas y las modas ideológicas sobre política económica, en la que se reedita periódicamente una promesa de prosperidad que, finalmente, no termina de llegar a todos.

A la crisis de la deuda externa de los años ochenta y los programas de austeridad fiscal les siguió el «Consenso de Washington» en los noventa, con la generalización de las medidas de liberalización interior y exterior (comercial y financiera), estabilidad macroeconómica, desregulación, privatización de empresas públicas y el $\triangleright$ 
agotamiento del modelo de crecimiento por sustitución de importaciones que sí logró transformarse y triunfar en algunos países del Sudeste Asiático. La siguiente década añadió el acento en los programas de lucha contra la pobreza y de promoción de la inversión en educación y sanidad, además del papel de las instituciones. Al desarrollo de China acompañó entonces un boom en las exportaciones de materias primas que vino a propiciar la década prodigiosa (2003-2013), en la que apareció una clase media no estrictamente capitalina y abrió una expectativa de prosperidad mejor compartida que hasta entonces. Ello se plasmó en caídas importantes en la desigualdad en una región que siempre ha tenido un reparto de la renta y la riqueza muy desigual. Posteriormente, el crecimiento fue debilitándose, pero se mantenía sin grandes desequilibrios en términos generales, lo que había dado lugar a una conversación propia de países emergentes sobre cómo aumentar la productividad desde un punto de vista microeconómico (tamaño empresarial, inversión en capital humano, calidad institucional y medidas de redistribución; en definitiva, cuestiones de medio plazo ligadas a la evolución de la productividad laboral). Este periodo se ha cerrado abruptamente con la crisis provocada por la pandemia del coronavirus.

A continuación se repasa la evolución y las previsiones para la región, tanto en cuanto a la renta como a la pobreza. Seguidamente se analiza el apoyo financiero concedido por el FMI a los países latinoamericanos en comparación con el resto del mundo y atendiendo a la casuística individual de los países del área iberoamericana. Por último se plantea la propuesta de crear un fondo especial, financiado voluntariamente con derechos especiales de giro (DEG), para financiar programas para países con elevada población bajo la línea de la pobreza y donde la desigualdad ha aumentado con la pandemia.

\section{Evolución y previsiones económicas}

En las dos últimas décadas, América Latina y el Caribe han experimentado una tasa de crecimiento superior a la de otros decenios, pero inferior a la mayoría del resto de regiones emergentes y en desarrollo. A partir de 2014, sin embargo, se observa un estancamiento del crecimiento de la región. La previsión del FMI (2021a) es que el conjunto de la región habrá pasado del crecimiento casi plano en 2019 al desplome de más del $7 \%$ en 2020 , con una expectativa de recuperación a tasas del 4\% en 2021 y algo menores en 2022 (Gráfico 2). La velocidad de vacunación durante estos meses y el daño estructural infringido al tejido productivo durante el periodo de contención de la epidemia determinarán si se cumplen estas previsiones.

Aunque la región de América Latina y el Caribe sólo cuenta con el $8,2 \%$ de la población mundial (640 millones de habitantes), a finales de septiembre de 2020 ya había registrado el $28 \%$ de los casos globales de infecciones por COVID (9,3 millones) y el $34 \%$ de los fallecimientos a causa de la enfermedad en todo el mundo (341.000 muertos). En el plano económico, las economías latinoamericanas presentan un mercado de trabajo más dependiente de sectores intensivos en contacto humano que la media de economías avanzadas, emergentes o de baja renta; sectores que, además, son menos susceptibles de adoptar formas telemáticas de ejecución y que emplean a trabajadores con menores niveles de renta o formación. Son también dependientes del turismo, las remesas y las exportaciones de materias primas (sobre todo en el Cono Sur), y las tres variables han tenido una pésima evolución como consecuencia de la pandemia.

A causa de los altos niveles de pobreza y de trabajo informal, y de la incapacidad de $D$ 
EL PAPEL DEL FONDO MONETARIO INTERNACIONAL EN AMÉRICA LATINA DURANTE LA CRISIS...
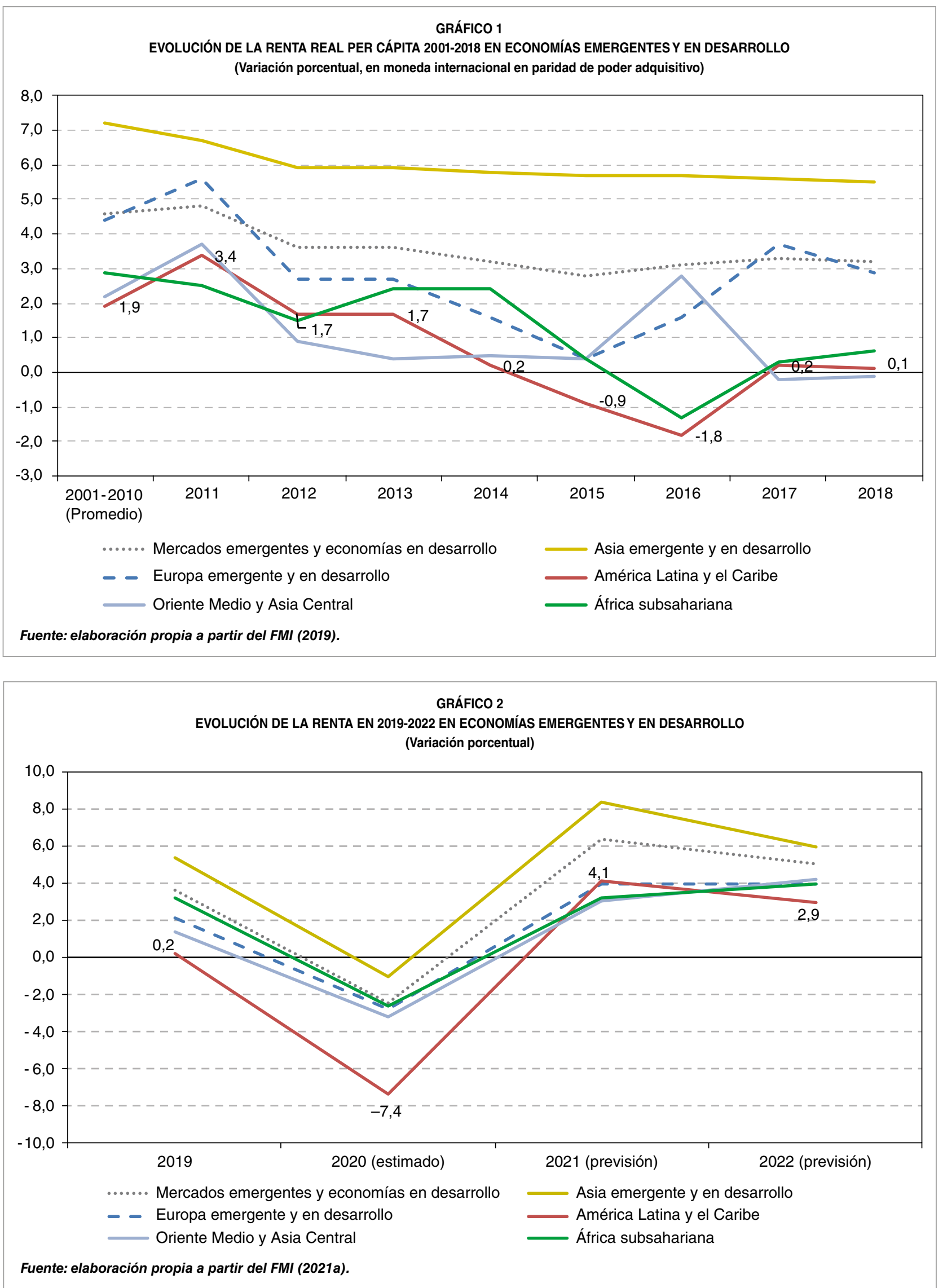
mantener distancias sociales en zonas urbanas densamente pobladas en muchas economías de la región, el levantamiento de las fuertes medidas de confinamiento impuestas en los inicios de la pandemia ha sido más costoso en ésta que en otras regiones del mundo con mayor peso de la industria (como Asia) o de mayor peso de un sector servicios de alto valor añadido que ha podido migrar al mundo online, como el de muchos países avanzados. A ello habría que añadir las menores capacidades sanitarias y de margen de maniobra fiscal con que cuentan muchos Gobiernos de la región (FMI, 2020a).

Precisamente, uno de los componentes que en América Latina requiere tanta o más atención que la velocidad de la recuperación de la renta es el impacto de la crisis sobre la pobreza y la desigualdad. La región, que ya era una de las más desiguales del mundo antes de la pandemia, va a experimentar un incremento significativo de las ratios de desigualdad y pobreza a consecuencia de la crisis del coronavirus, deshaciendo gran parte de los logros alcanzados en las décadas anteriores. Como se observa en el Cuadro 1, las mayores reducciones relativas de tasas de pobreza y número de personas pobres en la región se produjeron de 2002 a 2014, observándose una cierta ralentización a partir de entonces. En 2018, tanto las tasas de pobreza (22\% con menos de 5,50 dólares al día) como el número de personas bajo el umbral de pobreza (más de 140 millones con menos de 5,50 dólares al día) siguen siendo elevados.

Conforme a las estimaciones del FMI, el Banco Mundial y la CEPAL (Cuadro 2), la COVID-19 va a tener un impacto dramático en la pobreza. Por ejemplo, el FMI (2020b) estima un aumento de casi 15 millones de personas bajo el umbral de 1,90 dólares al día (lo cual supone un aumento relativo de más del $60 \%$ ). Asimismo, según el Informe de Panorama Social de América Latina de la CEPAL (CEPAL, 2021), debido a los efectos de la pandemia, y a pesar de las medidas de protección social para hacerle frente, los niveles de pobreza y pobreza extrema alcanzarán niveles no vistos en doce y veinte años, respectivamente, y se producirá un deterioro en la distribución de la renta en la mayoría de los países.

CUADRO 1

EVOLUCIÓN 1990-2018 DE LAS TASAS DE POBREZA Y NÚMERO DE PERSONAS BAJO EL UMBRAL DE LA POBREZA

\begin{tabular}{|c|c|c|c|c|c|c|c|c|c|c|c|c|c|c|}
\hline Indicador & $\begin{array}{l}\text { Umbral } \\
\text { pobreza }\end{array}$ & 1990 & 1993 & 1996 & 1999 & 2002 & 2005 & 2008 & 2011 & 2014 & 2015 & 2016 & 2017 & 2018 \\
\hline \multirow{3}{*}{ Tasas de pobreza (\%) } & 1,90 \$ día & 15,2 & 14,2 & 13,9 & 13,7 & 12,1 & 10,0 & 7,0 & 5,7 & 4,1 & 3,8 & 3,9 & 3,9 & 3,8 \\
\hline & 3,20 \$ día & 29,5 & 28,5 & 27,8 & 27,2 & 25,2 & 21,5 & 15,8 & 13,1 & 10,9 & 10,5 & 10,5 & 9,5 & 9,3 \\
\hline & 5,50 \$ día & 49,9 & 49,5 & 48,3 & 47,3 & 45,4 & 41,0 & 33,2 & 29,5 & 26,6 & 26,0 & 25,6 & 23,1 & 22,6 \\
\hline \multirow{3}{*}{$\begin{array}{l}\text { Número de personas en umbral } \\
\text { pobreza (millones) }\end{array}$} & 1,90 \$ día & 66,3 & 65,6 & 67,6 & 69,8 & 64,4 & 55,3 & 39,9 & 33,7 & 25,2 & 23,6 & 24,5 & 24,4 & 24,2 \\
\hline & 3,20 \$ día & 129,0 & 131,8 & 135,2 & 138,2 & 133,6 & 118,6 & 90,2 & 77,4 & 66,7 & 64,9 & 65,6 & 59,8 & 59,4 \\
\hline & 5,50 \$ día & 218,4 & 228,5 & 234,7 & 240,6 & 241,0 & 226,4 & 190,1 & 174,8 & 162,6 & 160,5 & 159,9 & 145,6 & 143,9 \\
\hline \multirow{3}{*}{$\begin{array}{l}\text { Tasas de variación de tasas de } \\
\text { pobreza }(\%)\end{array}$} & 1,90 \$ día & & $-7 \%$ & $-2 \%$ & $-1 \%$ & $-12 \%$ & $-17 \%$ & $-30 \%$ & $-19 \%$ & $-28 \%$ & & & $-5 \%$ & $-3 \%$ \\
\hline & 3,20 \$ día & & $-3 \%$ & $-2 \%$ & $-2 \%$ & $-7 \%$ & $-15 \%$ & $-27 \%$ & $-17 \%$ & $-17 \%$ & & & $-13 \%$ & $-2 \%$ \\
\hline & 5,50 \$ día & & $-1 \%$ & $-2 \%$ & $-2 \%$ & $-4 \%$ & $-10 \%$ & $-19 \%$ & $-11 \%$ & $-10 \%$ & & & $-13 \%$ & $-2 \%$ \\
\hline \multirow{3}{*}{$\begin{array}{l}\text { Tasas de variación de número } \\
\text { de personas en umbral pobreza } \\
(\%)\end{array}$} & 1,90 \$ día & & $-1 \%$ & $3 \%$ & $3 \%$ & $-8 \%$ & $-14 \%$ & $-28 \%$ & $-16 \%$ & $-25 \%$ & & & $-3 \%$ & $-1 \%$ \\
\hline & 3,20 \$ día & & $2 \%$ & $3 \%$ & $2 \%$ & $-3 \%$ & $-11 \%$ & $-24 \%$ & $-14 \%$ & $-14 \%$ & & & $-10 \%$ & $-1 \%$ \\
\hline & 5,50 \$ día & & $5 \%$ & $3 \%$ & $3 \%$ & $0 \%$ & $-6 \%$ & $-16 \%$ & $-8 \%$ & $-7 \%$ & & & $-10 \%$ & $-1 \%$ \\
\hline
\end{tabular}


CUADRO 2

IMPACTO ESTIMADO DE COVID-19 EN LA POBREZA EN AMÉRICA LATINA Y EL CARIBE

(Millones de personas)

\begin{tabular}{|c|c|c|c|c|}
\hline Fuente & $\begin{array}{l}\text { Umbral } \\
\text { pobreza }\end{array}$ & $\begin{array}{l}\text { Aumento estimado } \\
\text { de personas bajo el } \\
\text { umbral de pobreza } \\
\text { por COVID-19 } \\
\text { (millones) (C) }\end{array}$ & $\begin{array}{c}\text { Tasa de variación del número } \\
\text { de personas bajo el umbral } \\
\text { de pobreza por COVID-19 } \\
\text { estimado en relación con } \\
\text { base } 2018 \\
(\%)(\mathrm{C} / \mathrm{E})^{\star} 100\end{array}$ & $\begin{array}{l}\text { Número de personas bajo } \\
\text { el umbral de la pobreza } \\
\text { en } 2018 \\
\text { (millones) } \\
\text { (E) }\end{array}$ \\
\hline Fiscal Monitor (octubre 2020) & 1,90 \$ día & 14,7 & $61,00 \%$ & 24,2 \\
\hline Banco Mundial (junio 2020) & 5,50 \$ día & $18-35$ & $18,42 \% *$ & 143,9 \\
\hline CEPAL (julio 2020) & 5,50 \$ día & 44 & $31,00 \%$ & 143,9 \\
\hline
\end{tabular}

\section{Programas del Fondo Monetario Internacional en América Latina}

La nueva financiación a países miembros del FMI, aprobada desde marzo de 2020 hasta el momento de la redacción de este artículo (comienzos de marzo de 2021), cubre una espectacular lista de 85 países y supera los 100.000 millones de dólares (77.666 millones de DEG)1. La mayor parte (más de 72.000 millones) se ha destinado a financiar programas tradicionales ${ }^{2}$, bien con desembolsos programados, bien con precautorios. El resto (algo más de 30.000 millones) ha permitido financiar programas de financiación de emergencia, en sus dos modalidades: i) instrumentos de financiamiento rápido, destinados fundamentalmente a países de renta media; y ii) servicios de crédito rápido, destinados a países de baja renta en términos concesionales. Ambos tipos de programa permiten suministrar asistencia financiera rápida a todos los países miembros que enfrentan una necesidad urgente de

1 Fuente: FMI, página web de asistencia financiera aprobada frente a la COVID-19. (https://www.imf.org/en/Topics/imf-and-covid19/COVIDLending-Tracker). A 5 de marzo, el tipo de cambio dólar-DEG es de 1,42 dólares estadounidenses por DEG.

2 Entendiendo por «programa tradicional» cuatro tipos de programa, que incluyen los de desembolsos programados y las líneas precautorias: los Acuerdos Stand-By (SBA), el Servicio Ampliado del FMI (EFF), la Línea de Crédito Flexible (LCF) y la Línea de Precaución y Liquidez (LPL). balanza de pagos; ninguno de los dos plantea una estructura de condicionalidad ex post, si bien pueden incluir medidas previas (prior actions). Adicionalmente, los países de baja renta se han visto beneficiados por el Fondo Fiduciario para Alivio y Contención de Catástrofes, que permite asistir a los países pobres que se enfrentan a desastres de salud pública —como epidemias o pandemias - con donaciones para el alivio del servicio de la deuda.

Como se observa en el Gráfico 4, más del $60 \%$ de los programas aprobados durante la pandemia se dirigen a países iberoamericanos. Entre ellos, la gran mayoría (casi 68.000 millones de dólares) se destinan a países iberoamericanos $^{3}$. Los programas de las islas del Caribe, que, a grandes rasgos, son los que no forman parte de la comunidad iberoamericana, requieren escasa cuantía. Entre los programas aprobados para América Latina, solo algo más de 3.600 millones de DEG se destinan a financiación de emergencia (Gráfico 5). La gran mayoría, por tanto, son programas tradicionales (con o sin desembolsos programados).

3 Países de la comunidad iberoamericana: Andorra, Argentina Bolivia, Brasil, Colombia, Costa Rica, Cuba, Chile, República Dominicana, Ecuador, El Salvador, España, Guatemala, Honduras, México, Nicaragua, Panamá, Paraguay, Perú, Portugal, Uruguay y Venezuela. 

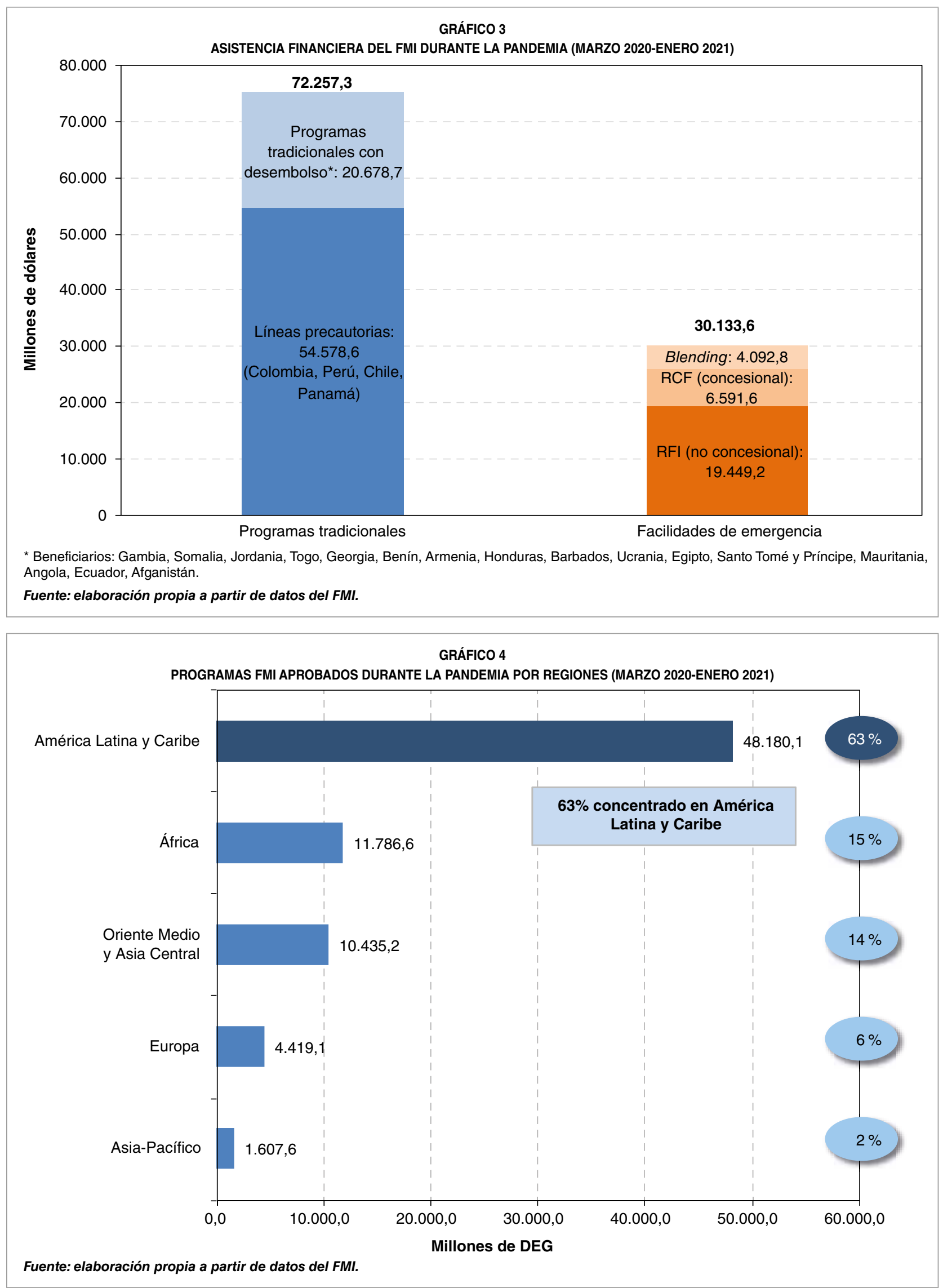


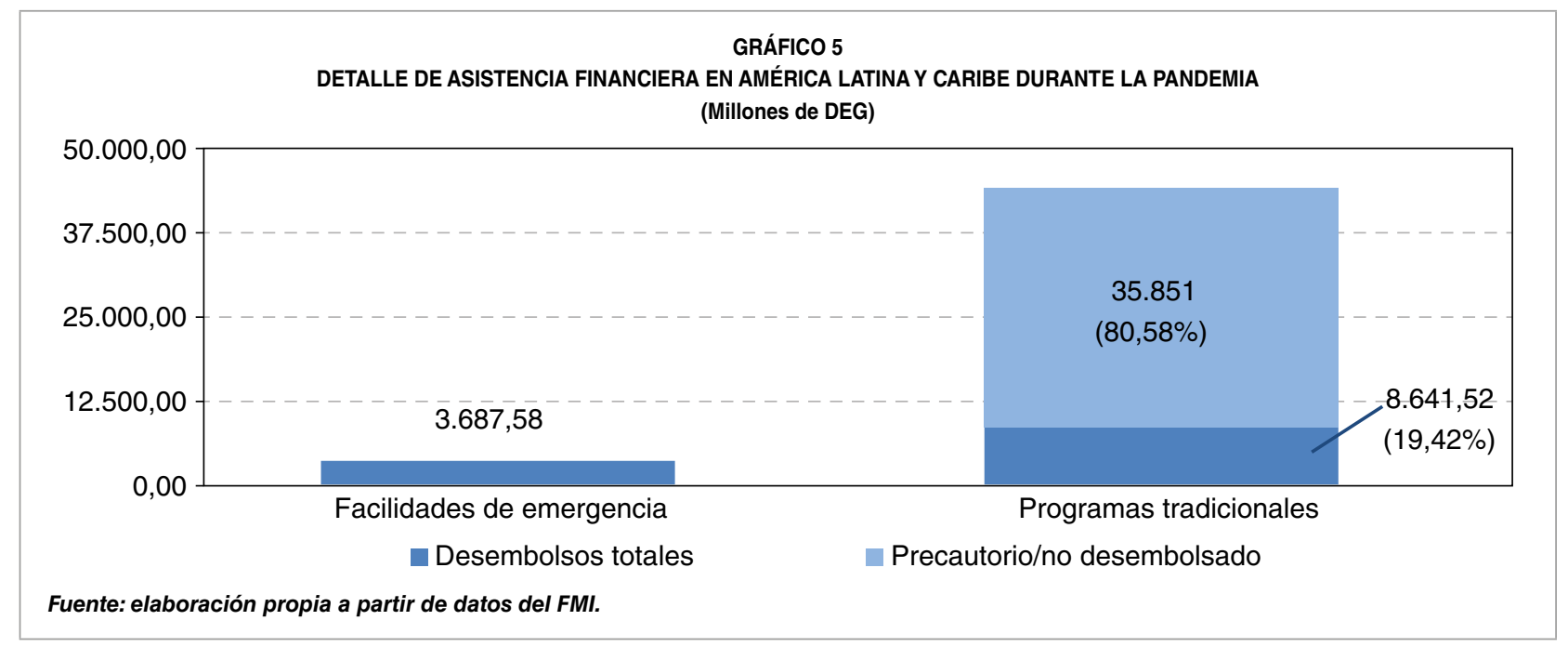

Entre los programas de financiación no concesionales, casi el $80 \%$ de la financiación comprometida (incluyendo compromisos previos a la pandemia) se dedica a América Latina. La mayor cuantía corresponde a líneas de apoyo precautorias, es decir, donde no hay desembolsos programados. Los desembolsos, en realidad, suponen solo el $46 \%$, en comparación con el $84 \%$ que suponen los compromisos de crédito a los países latinoamericanos bajo la Cuenta General de Recursos (GRA), que suministra financiación para los programas no concesionales.

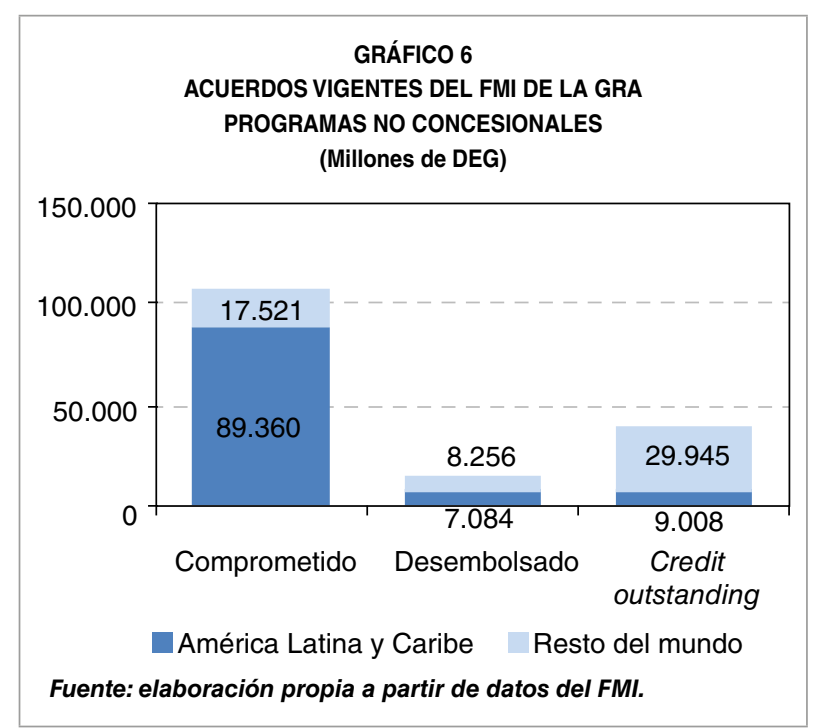

Pasando al crédito pendiente, la exposición del FMI a América Latina supera el $40 \%$ (cerca de 44.000 millones de DEG). En el resto del mundo el FMl tiene una exposición del 59\% (cerca de 62.000 millones de DEG; Gráfico 8). En esta exposición, los principales deudores del FMI son Argentina, Egipto, Ucrania, Pakistán y Ecuador, en este orden (Gráfico 9). Dos de los cinco mayores deudores del Fondo son iberoamericanos, y suponen un $39 \%$ del crédito pendiente. Argentina supone por sí sola un $34 \%$ de la exposición de la cuenta general de recursos del FMI (31.900 millones de DEG).

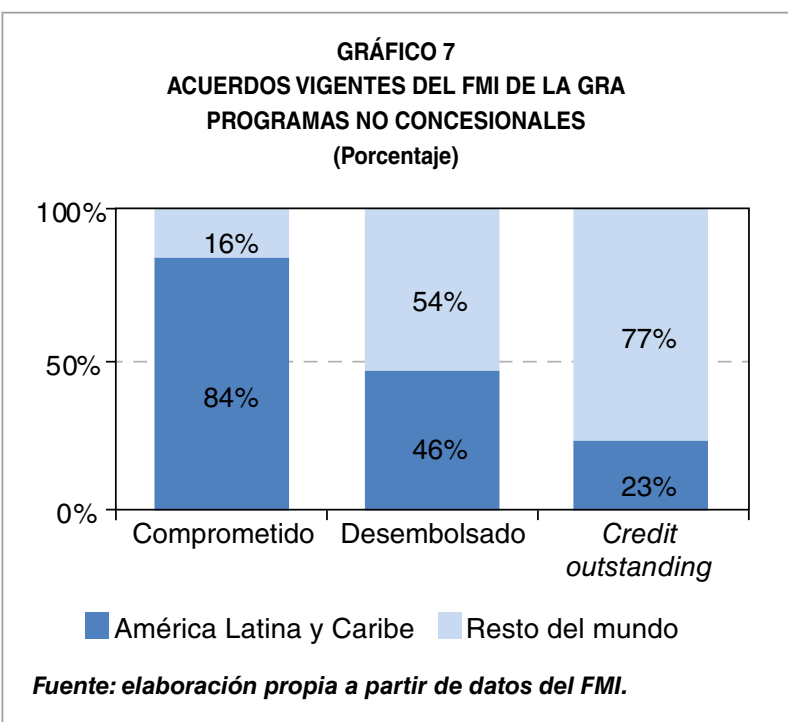



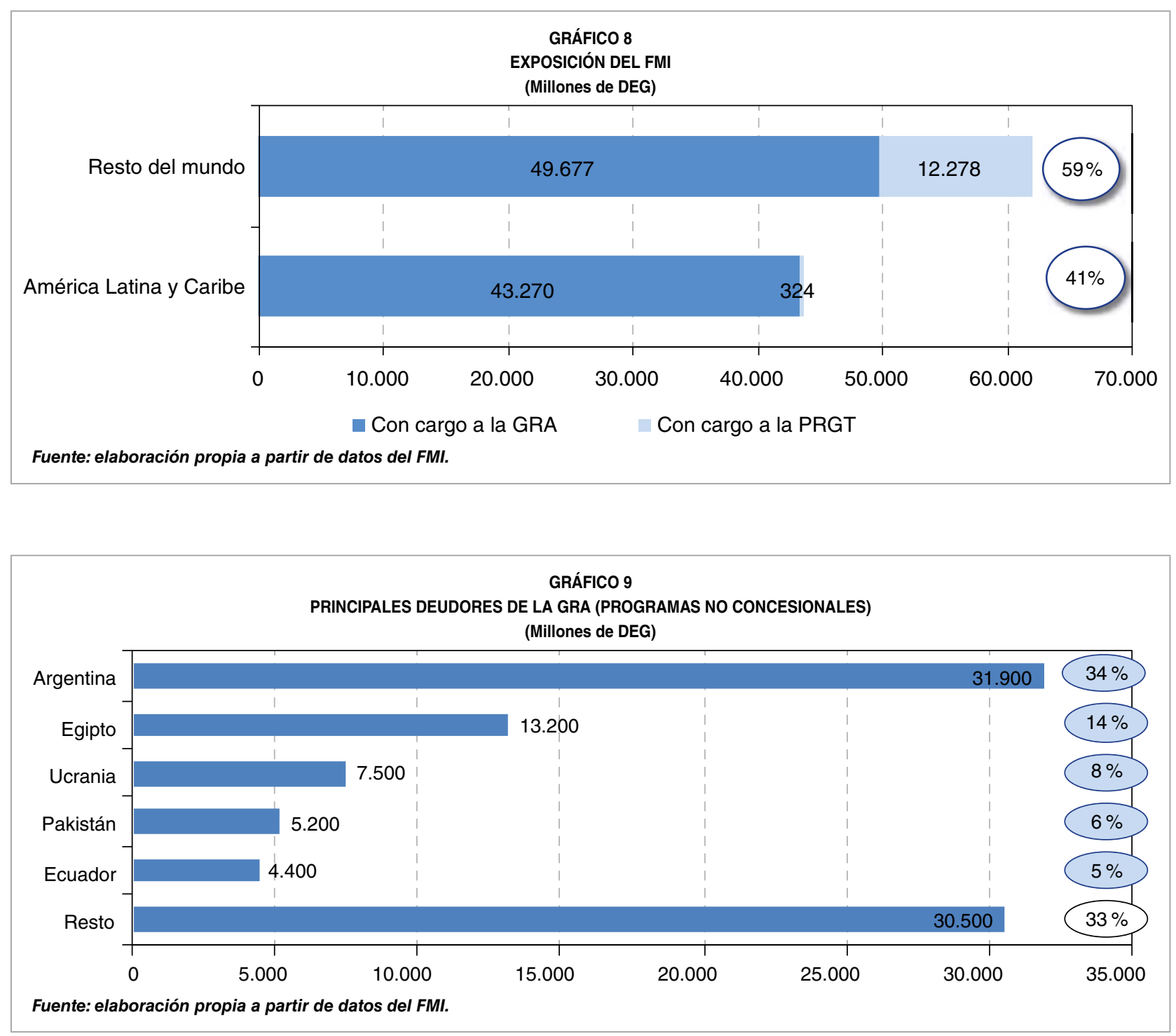

En febrero de 2021, la mayoría de los países iberoamericanos que son miembros del FMI (todos salvo Cuba, que no es miembro) cuenta con un programa activo. Las excepciones son Brasil, Venezuela, Uruguay y Argentina. Este último no tiene un programa activo, pero es el principal deudor del Fondo y está negociando uno nuevo.

A grandes rasgos, el tipo de programas refleja no solo las necesidades, sino también la solidez macroeconómica y de políticas del país. Cuatro de los grandes países tienen líneas de crédito flexible (LCF), programas de naturaleza precautoria otorgados a países que presenten sólidos fundamentos macroeconómicos ex ante, así como un marco de política económica e historial en la aplicación de políticas muy sólidos. Por lo tanto, la aprobación de estos programas supone un espaldarazo al marco de políticas macroeconómicas de los respectivos Gobiernos; son los países de la costa del Pacífico, salvo Ecuador. El de México es el más cuantioso (44.564 millones de DEG) y se aprobó antes del estallido de la pandemia. Le siguen los de Colombia (12.267 millones de DEG, de los cuales se han desembolsado $D$ 


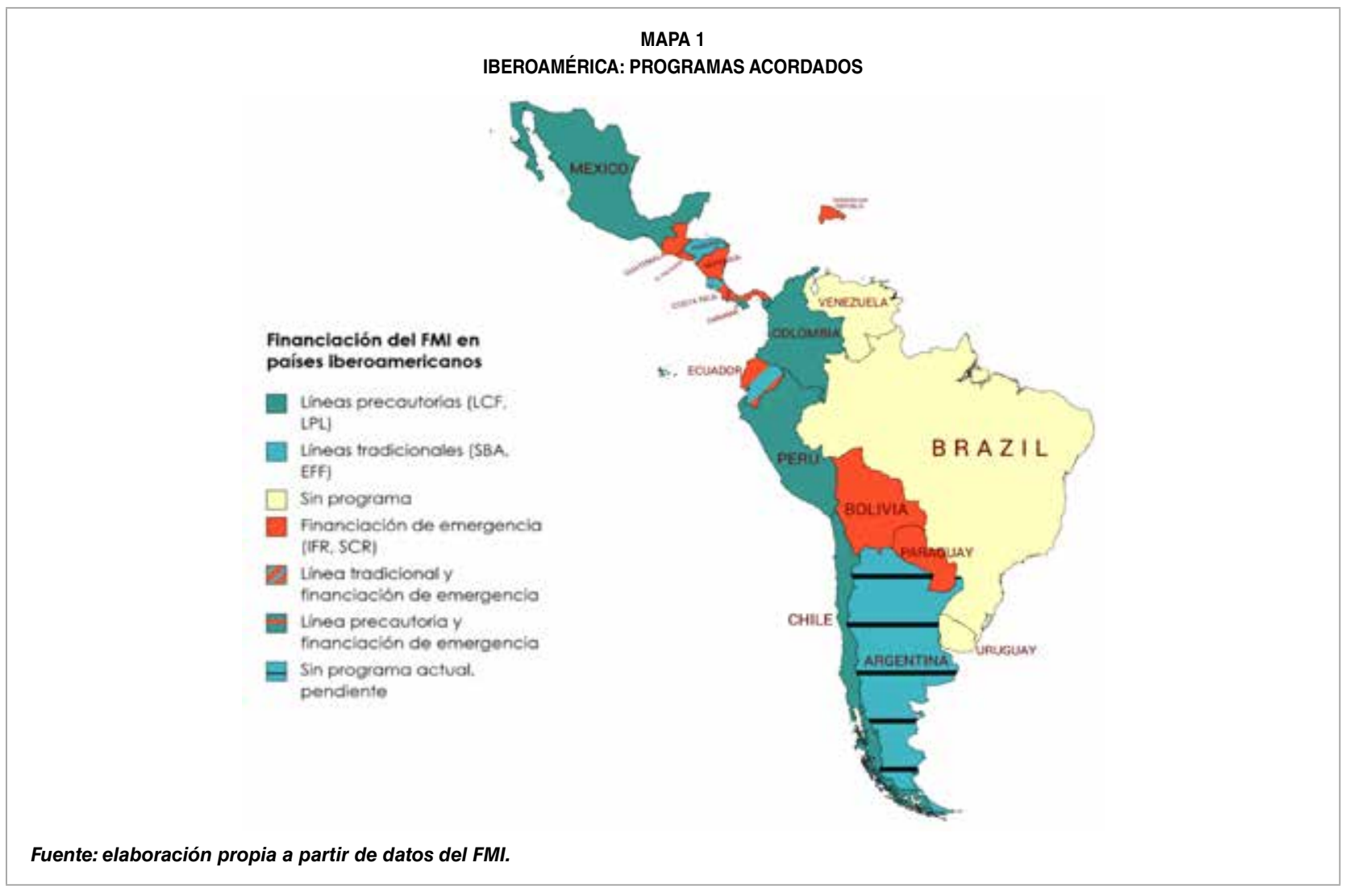

3.750 millones, y que es una reedición de programas anteriores), Perú (8.007 millones de DEG) y Chile (17.443 millones de DEG). Por otra parte, se aprobó a principios de 2021 a favor de Panamá una línea precautoria y de liquidez (LPL) (1.884 millones de DEG), programa también de naturaleza precautoria que permite satisfacer las necesidades de liquidez de los países miembros con sólidos fundamentos económicos y sólidos antecedentes de implementación de políticas, pero con algunas vulnerabilidades macroeconómicas.

Un tercer grupo es el compuesto por países que cuentan con programas tradicionales SBA (Acuerdo Stand-By) o EFF (Servicio Ampliado del FMI) que o bien son previos a la crisis o bien han sido reeditados o están en proceso de reedición. En primer lugar, Argentina es el país con mayor deuda frente al FMI, con un crédito pendiente de 31.914 millones de DEG, correspondientes a un SBA aprobado en junio de 2018; en la actualidad, Argentina y el FMI negocian un posible nuevo acuerdo. En segundo lugar, Ecuador, que ostenta la quinta mayor deuda frente al FMI, alcanzó un acuerdo de Servicio Ampliado a finales de 2020 (4.615 millones de DEG). A ellos cabe sumar a Costa Rica, que en marzo de $2021^{4}$ ha alcanzado un acuerdo de Servicio Ampliado por valor de 1.778 millones de DEG. Finalmente, Honduras ha ampliado un programa Stand-By preexistente (financiado en parte de forma concesional).

En cuanto a los programas de financiación de emergencia, como países de renta media, la mayor parte de los países iberoamericanos que han solicitado esta modalidad ha optado por un Instrumento de Financiamiento $\square$

4 Por razón de distinta fecha de corte, el reciente EFF concedido a Costa Rica no figura en los cuadros. 


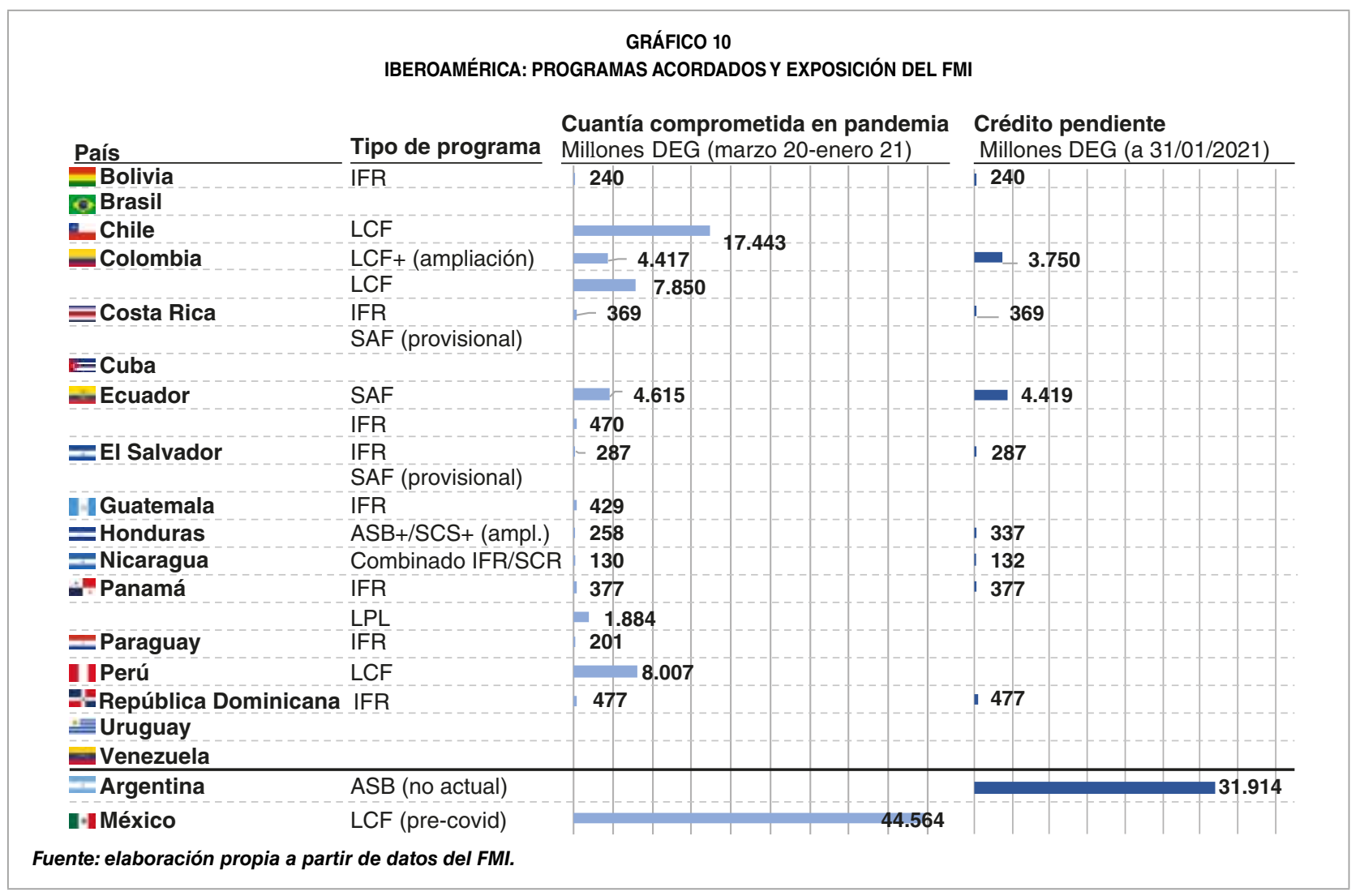

Rápido: Bolivia, Costa Rica, Ecuador, El Salvador, Guatemala, Nicaragua (conjunto con Servicio de Crédito Rápido), Panamá, Paraguay y República Dominicana. En general, estos programas han permitido desplegar financiación de emergencia por volumen del $100 \%$ de las respectivas cuotas de estos países en el Fondo.

En definitiva, de los países iberoamericanos grandes, todos, salvo Venezuela, Uruguay y Brasil, tienen programa activo con el FMI o están negociándolo, lo cual aumentará la exposición del Fondo a América Latina, que ya es elevada (la más elevada en comparación con otras regiones). Esta observación lleva a plantearse qué más se puede hacer para facilitar una salida de la crisis de la región.

\section{4. ¿Qué más se puede hacer?}

Tras la caída de Lehman Brothers en 2008, la membresía del FMI decidió aprobar una asignación general por valor de 250.000 millones de dólares. Los DEG son un activo potencial contra monedas de reserva internacional y computan como reservas de los bancos centrales. Una asignación general equivale a un aumento de liquidez mundial, permitiendo a los países de divisa débil lograr acceso a financiación en divisa fuerte, lo que facilita la financiación de los países que los reciben. En el ámbito del sistema monetario internacional, una de las medidas más contundentes frente a la Gran Recesión fue llevar a cabo la mayor asignación general de la historia, como se observa en el Gráfico 11.

En la actualidad, los miembros del FMI se han venido planteando nuevamente la $D$ 


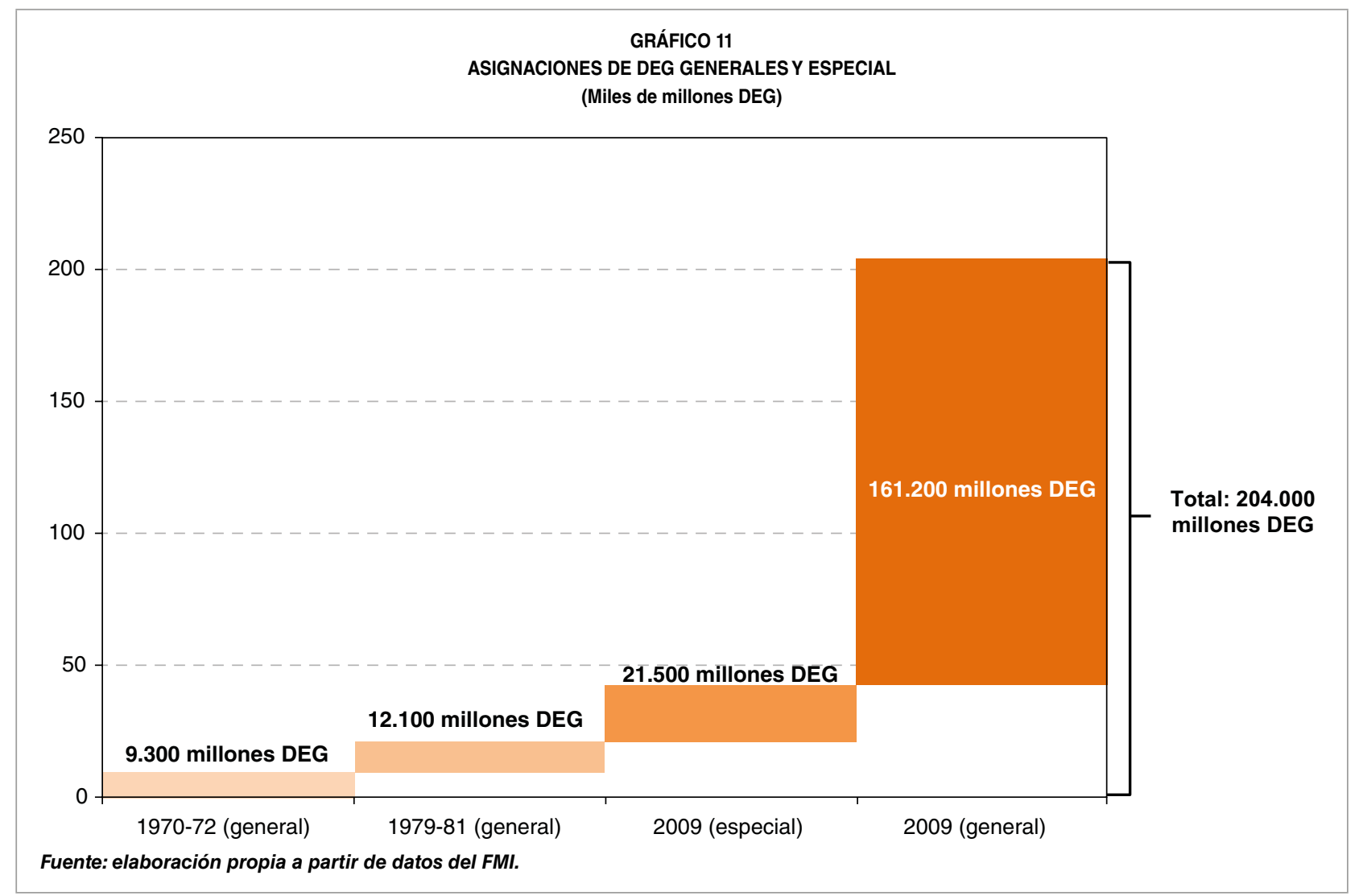

posibilidad de aprobar una asignación general de DEG. El voto favorable de Estados Unidos es necesario en esta decisión debido al tamaño de su cuota en el Fondo y el porcentaje de votos favorables necesario para adoptarla. Aunque no existe consenso sobre el volumen de una hipotética asignación, cabe recordar que en los inicios de la crisis se planteó un volumen de unos 350.000 millones de DEG. Esta cifra es especialmente relevante por un motivo: supondría una asignación general tal que a Estados Unidos le correspondería una cantidad de DEG inferior a su cuota en el Fondo, circunstancia que permite al Tesoro de Estados Unidos votar a favor de la medida sin necesidad de requerir aprobación parlamentaria previa.

En una asignación general los DEG se distribuyen a los países de acuerdo con sus cuotas en el FMI. Ahora bien, dado que los países desarrollados son los que mayor cuota ostentan en conjunto, una parte sustancial de los nuevos DEG irían a reforzar los balances de bancos centrales que emiten moneda fuerte. Por ejemplo, las cuotas sumadas de Estados Unidos, Reino Unido, la zona euro y Japón suponen más del $50 \%$ del total (sin contar con el resto de economías avanzadas). En consecuencia, en el caso de una asignación general de 350.000 millones de DEG, corresponderá a sus bancos centrales unos 21.500 millones, que previsiblemente no van a utilizar para obtener divisas. Por el contrario, las economías emergentes y en desarrollo, las más necesitadas de acceso a divisa fuerte, percibirían en torno al $42 \%$ de los nuevos DEG, de los cuales solo alrededor del $3 \%$ sería distribuido a economías de baja renta.

En este contexto, cabe la posibilidad de que los bancos centrales de los países con una sólida posición externa presten al FMI los DEG $\triangleright$ 
que no necesitan mantener en sus reservas (en el caso de los países de la zona euro, con ciertos límites). Este mecanismo de reciclaje de DEG ociosos se utiliza ya para financiar un fondo fiduciario en el FMl que otorga préstamos concesionales a los países más pobres (el Fondo Fiduciario para el Crecimiento y la Reducción de la Pobreza, o PRGT). Sin embargo, no existen mecanismos similares para mejorar el acceso a financiación para países de renta media.

Así las cosas, se puede plantear la creación de un fondo fiduciario financiado voluntariamente con los DEG excedentarios de países cuya posición externa sea sólida, al igual que se financia voluntariamente el destinado a programas concesionales para países de renta baja. Este nuevo fondo otorgaría préstamos supervisados por el personal del FMI, que serían complementarios a los programas convencionales existentes. Se podría dar acceso a esta financiación a países que, siendo de renta media, tuvieran ya antes de la pandemia bolsas de pobreza y un problema de desigualdad y en los que la pandemia haya empeorado la situación de desequilibrio social. Además, los fondos se podrían dedicar a fomentar la inversión pública para mitigar el cambio climático. Los préstamos se concederían al tipo de interés de los DEG, lo cual supone una ventaja con respecto al precio de los préstamos tradicionales.

\section{Conclusiones}

Desde el comienzo de la crisis provocada por las medidas de contención de la pandemia del coronavirus, el FMI ha aprobado programas de apoyo financiero por valor de más de 100.000 millones de dólares para 85 países. Una parte se ha dedicado a financiación de emergencia, pero la mayor parte del monto total se ha destinado a unos pocos programas tradicionales, bien precautorios, bien con desembolsos programados. América Latina es la región a la que el FMI está más expuesto (aunque cuenta solo con un $23 \%$ del crédito pendiente de los acuerdos vigentes del Fondo de la GRA) y la región en la que hay ya comprometido más apoyo financiero bajo los programas no concesionales (el $80 \%$ ), incluyendo compromisos previos a la crisis del coronavirus. Esta diferencia se debe al elevado peso de unas pocas líneas precautorias para países de la región.

Se vislumbra el final de la crisis a medida que se vayan distribuyendo las vacunas los próximos meses, pero queda la cuestión de qué grado de daño habrá sufrido el tejido productivo y social de estas economías y cómo fomentar la recuperación mediante el impulso de la demanda agregada y la reorientación hacia un modelo económico más verde y digital. Al igual que sucede en los países desarrollados, está en manos de los Gobiernos poner las condiciones para que en la próxima década no se repita la situación de inversión pública deprimida que siguió a la crisis de la zona euro en varios países. Simultáneamente, la inversión pública debe centrarse en promover los cambios necesarios para hacer frente a la emergencia climática. Por último, el impacto de la crisis en la pobreza y la desigualdad es dramático. La experiencia histórica muestra que, en ausencia de medidas, en pocos años se puede reflejar en inestabilidad política.

Por estas razones, hay que plantearse si, más allá de la provisión de financiación de emergencia y de líneas precautorias para que los Gobiernos mantengan sus políticas actuales, el FMl debe contribuir al esfuerzo para la recuperación en países de renta media, incluyendo América Latina. La región ya sufrió una década perdida tras la crisis de la deuda de 1982. Se trata de evitar que ahora sufra otra. $\triangleright$ 


\section{Bibliografía}

Banco Mundial (2020). Poverty and shared prosperity 2020: Reversals of Fortune. Washington, DC: World Bank. Poverty and Shared Prosperity 2020 (worldbank.org)

Comisión Económica para América Latina y el Caribe (2021). Panorama Social de América Latina 2020. https://www.cepal.org/es/publicaciones/ 46687-panorama-social-america-latina-2020

Fondo Monetario Internacional (2019). World Economic Outlook: Global Manufacturing Downturn, Rising Trade Barriers. Washington, DC, octubre de 2019. World Economic Outlook (imf.org).

Fondo Monetario Internacional (2020a). Monitor Fiscal: Octubre de 2020. https://www.imf.org/es/ Publications/FM/Issues/2020/09/30/october-2020fiscal-monitor

Fondo Monetario Internacional (2020b). Western Hemisphere. Pandemic Persistence Clouds the
Recovery. Regional Economic Outlook, octubre de 2020. https://www.imf.org/en/Publications/ REO/WH/Issues/2020/10/13/regional-economicoutlook-western-hemisphere

Fondo Monetario Internacional (2021a). World Economic Outlook Update: Policy Support and Vaccines Expected to Lift Activity. World Economic Outlook Update, January 2021: Policy Support and Vaccines Expected to Lift Activity (imf. org).

Fondo Monetario Internacional (2021b). IMF Credit Outstanding for all members as of January 31, 2021. https://www.imf.org/external/np/fin/tad/extcred2.aspx?date $1 \mathrm{key}=2021-01-31$ \& reportdate $=2021-01-31$

Fondo Monetario Internacional (2021c). Weekly Report on Key Financial Statistics, 5 de febrero de 2021. https://www.imf.org/external/np/tre/activity/2021/020521.pdf 
